Research Article

\title{
Sliding Mode Integrated Control for Vehicle Systems Based on AFS and DYC
}

\author{
Chao Lu $(\mathbb{D}$, Jing Yuan $(\mathbb{D}$, and Genlong Zha \\ School of Information Engineering, Suqian University, Suqian 223800, China \\ Correspondence should be addressed to Chao Lu; luxiaochao6@163.com
}

Received 22 September 2020; Revised 30 October 2020; Accepted 16 November 2020; Published 10 December 2020

Academic Editor: Shihong Ding

Copyright (c) 2020 Chao Lu et al. This is an open access article distributed under the Creative Commons Attribution License, which permits unrestricted use, distribution, and reproduction in any medium, provided the original work is properly cited.

\begin{abstract}
This paper has investigated an integrated control of active front steering (AFS) and direct yaw-moment control (DYC) for vehicle systems. First of all, the desired yaw rate and sideslip angle are estimated by using a two-degree-of-freedom (2-DOF) model of the vehicle system. On this basis, the actual sideslip angle is estimated by means of an observer. Then, the sliding mode control (SMC) is developed for AFS and DYC, respectively, to guarantee that the actual yaw rate and the sideslip angle track their reference signals. Additionally, the disturbance observer (DOB) technique is introduced to further improve the control performance. Finally, the simulation results validate the superiority of the AFS and DYC integrated control by using CarSim software during the following conditions: double lane change and side wind disturbance.
\end{abstract}

\section{Introduction}

With the development of electronic technology, the use of control technology to improve the active safety of automobiles has become a hot topic in the automotive field. Active safety can minimize or avoid traffic accidents through vehicle design, while passive safety means that vehicles are designed to minimize the damage to passengers after an accident occurs. In the 90s of the last century, the concept of vehicle stability was proposed [1-3]. Then, the active safety control received considerable attention in the vehicle stability control, such as [4-7]. Among them, AFS catches researchers' eyes since it can directly adjust the steering angle of the drive. Nevertheless, when the moment of the vehicle in control limit is under the sections of high-speed turning, heavy braking, or acceleration, steering will have no effect or limited effect, the car loses the ability to turn, and the initiative will have little effect on the steering. On the contrary, the effect of DYC is very obvious at this time, and it is easy to realize. It does not require a great change in the original structure of the vehicle. This is because the goal of DYC is to adjust the vehicle yaw motion. Thus, the integrated control of AFS and DYC can not only further improve the lateral stability of the vehicle but also reduce the influence of braking on longitudinal dynamics and improve the driving comfort.
In [7], the LQR method was used in the integrated control of AFS and DYC and compared with DYC control. In [8], an integrated control of AFS and DYC with forward and feedback controllers was presented, and the parameters of the feedback controller were obtained by the optimum control theory. Then, the control strategy of AFS based on sliding mode theory and the control strategy of EPS (combined control on both direct yaw moment and variable slip ratio) were presented in [9]. An integrated AFS and DYC control system was developed in [10] based on the fuzzy logic control, which was used for the yaw rate controller to keep the yaw rate in its ideal value. In [11], the model predictive control was used to adopt the hierarchical integrated control structure.

In addition, on the one hand, the sliding mode control (SMC) is popular for rejecting the uncertainties [12-18]. On the other hand, the accurate mathematical model is indeed necessary for SMC $[19,20]$. Hence, it is obvious that the SMC method is a very useful tool for active safety control, such as [21-23].

This paper focuses on the investigation of the integrated SMC control for the AFS and DYC system, which is designed to make sure the actual yaw rate and sideslip angle track the desired signals. First of all, the proposed control can drive 
the error of the yaw rate and the sideslip angle to zero within a finite time. Then, a disturbance observer (DOB) [24] is constructed for the proposed control to reduce the control gain so as to reduce the chattering. The effectiveness of the proposed control is illustrated by the simulation on MATLAB and CarSim.

As compared with the existing results, the contributions of this paper are twofold. On the one hand, an integrated control of AFS and DYC for vehicle systems has been studied. On the other hand, the Lyapunov stability analysis and simulation results have been given to demonstrate the effectiveness of the proposed strategy.

The rest of the paper is organized as follows. Section 2 introduces the dynamic model of the vehicle and problem statement. The process of control design is given in Section 3. The simulation results are shown in Section 4. Section 5 concludes this paper.

\section{Dynamical Model and Problem Statement}

2.1.2-DOF Model. The 2-DOF model is called as the "bicycle model" $[25,26]$, which is shown in Figure 1.

Then, the model is described as

$$
\begin{aligned}
m V_{x}(\dot{\beta}+r)= & -2\left(C_{f}+C_{r}\right) \beta+\frac{-2\left(a C_{f}-b C_{r}\right)}{V_{x}} r+2 C_{f} \delta_{f} \\
I_{z} \dot{r}= & -2\left(a C_{f}-b C_{r}\right) \beta+\frac{-2\left(a^{2} C_{f}+b^{2} C_{r}\right)}{V_{x}} r \\
& +2 a C_{f} \delta_{f},
\end{aligned}
$$

where $C_{f}$ and $C_{r}$, respectively, are the front and rear tire cornering stiffness, $F$ is the tire force, $x$ means the longitudinal position, $y$ means the lateral position, $\beta$ is the sideslip angle, $a$ and $b$ mean the distances from the center of gravity to the front and rear axles, $V$ is the velocity, $f$ means "front," $r$ means "rear," $r$ is the yaw rate, $I_{z}$ is the moment of inertia, $\delta_{f}$ is the front-wheel steering angle, and $m$ is the mass.

2.2. Problem Formulation. It is important to make sure what the ideal reference signal is. Usually, the yaw rate and the sideslip angle are the two important parameters to measure vehicle stability. According to the 2-DOF model, the desired yaw rate and sideslip angle can be calculated as [27]

$$
\begin{aligned}
& r_{d}= \begin{cases}r_{t}, & \left|r_{t}\right|<\frac{0.85 \mu g}{V_{x}}, \\
\frac{0.85 \mu g}{V_{x}} \operatorname{sign}\left(r_{t}\right), & \left|r_{t}\right| \geq \frac{0.85 \mu g}{V_{x}},\end{cases} \\
& \beta_{d}= \begin{cases}\beta_{t}, & \left|\beta_{t}\right|<\beta_{\max }, \\
\beta_{\max } \operatorname{sign}\left(\beta_{t}\right), & \left|\beta_{t}\right| \geq \beta_{\max },\end{cases}
\end{aligned}
$$

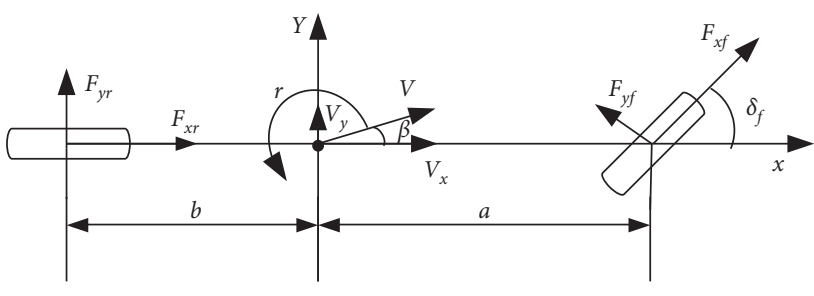

Figure 1: 2-DOF vehicle model.

with

$$
\begin{aligned}
& r_{t}=\frac{V_{x}}{(a+b)\left(1+K V_{x}^{2}\right)} \delta_{f d}, \\
& \beta_{t}=\frac{b-a m V_{x}^{2} / 2 C_{f}(a+b)}{(a+b)\left(1+K V_{x}^{2}\right)} \delta_{f d},
\end{aligned}
$$

where $\mu$ is the tire-road friction coefficient, $K$ is a positive constant, $\delta_{f d}$ is the angle input from the steering wheel to the front wheel, $\beta_{\max }=\arctan (0.02 \mu \mathrm{g})$, and $g$ is the gravitational constant.

The diagram of the integrated control of active front steering and direct yaw moment (AFS + DYC) is depicted in Figure 2, and the vehicle inputs include the vehicle speed $V_{x}$ and the steering wheel angle $\delta_{f d}$ commanded by the driver. According to the 2-DOF vehicle model, the ideal sideslip angle $\beta_{d}$ and yaw rate $r_{d}$ can be calculated, respectively. The CarSim vehicle model is regarded as a real vehicle model, which can output the actual yaw rate $r$. As a matter of fact, it is difficult to measure the actual sideslip angle directly by some special sensors, and the sensors needed are expensive. On the contrary, the accuracy and stability need to be further improved, thus designing the state observer to estimate the exact value is a good solution.

Remark 1. The DYC algorithm cannot be imposed on the 2 -DOF model. This is because the implementation of DYC algorithm is based on the yaw moment $M_{z}$ generated by the torque difference between left and right wheels, while the 2-DOF model regards the left and right wheels as one wheel such that there is no $M_{z}$ in the 2-DOF model.

\section{Control Design}

3.1. Sideslip Angle Observer. The lateral acceleration $a_{y}$ is expressed as

$$
a_{y}=V_{x}(\dot{\beta}+t r)=\frac{-2\left(C_{f}+C_{r}\right)}{m} \beta+\frac{-2\left(a C_{f}-b C_{r}\right)}{m V_{x}} r+\frac{2 C_{f}}{m} \delta_{f} .
$$

Introduce the variables $x_{1}=r, x_{2}=\beta, X=[r, \beta]^{T}$, $Y=\left[y_{1}, y_{2}\right]^{T}=\left[r, a_{y}\right]^{T}$, and $u=\left[\delta_{f}\right]$. The vehicle model described by equations (1) and (2) can be rewritten as 


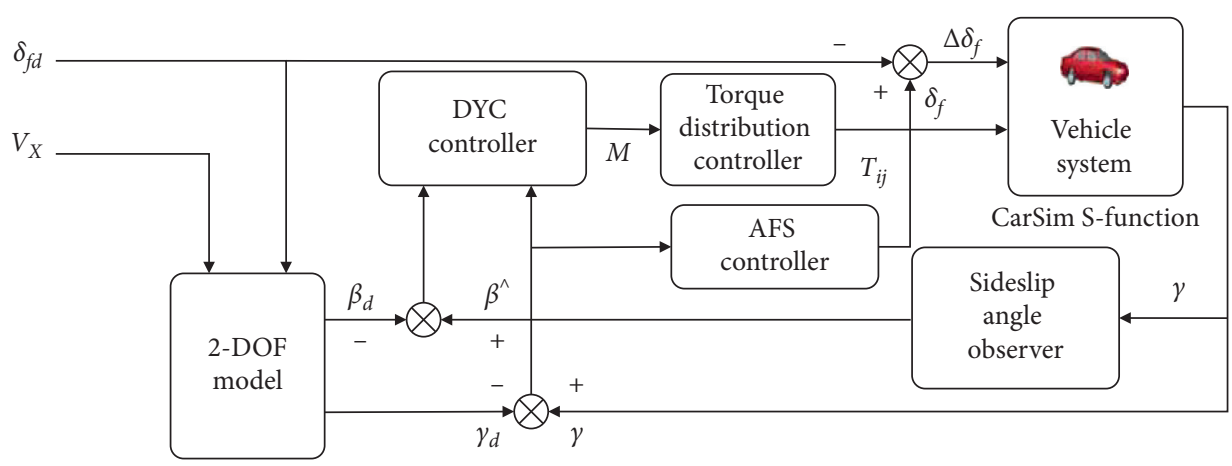

FIgURE 2: Structure of the AFS + DYC control system.

$$
\begin{aligned}
& \left\{\begin{array}{l}
\dot{X}=A X+B u, \\
Y=C X+D u
\end{array}\right. \\
& A=\left[\begin{array}{cc}
\frac{-2\left(a^{2} C_{f}+b^{2} C_{r}\right)}{I_{z} V_{x}} & \frac{-2\left(a C_{f}-b C_{r}\right)}{I_{z}} \\
\frac{-2\left(a C_{f}-b C_{r}\right)}{m V_{x}^{2}}-1 & \frac{-2\left(C_{f}+C_{r}\right)}{m V_{x}}
\end{array}\right], B=\left[\begin{array}{c}
\frac{2 a C_{f}}{I_{z}} \\
\frac{2 C_{f}}{m V_{x}}
\end{array}\right], \\
& C=\left[\begin{array}{cc}
1 & 0 \\
V_{x}\left(A_{21}+1\right) & V_{x} A_{22}
\end{array}\right], \quad D=\left[\begin{array}{c}
0 \\
V_{x} B_{2}
\end{array}\right] \text {. }
\end{aligned}
$$

Then, the observer is constructed as

$$
\left\{\begin{array}{l}
\dot{\hat{x}}_{1}=A_{11} y_{1}+A_{12} \hat{x}_{2}+B_{1} u+c_{1}\left|y_{1}-\hat{x}_{1}\right|^{1 / 2} \operatorname{sign}\left(y_{1}-\hat{x}_{1}\right), \\
\dot{\hat{x}}_{2}=A_{21} y_{1}+A_{22} \hat{x}_{2}+B_{2} u+c_{2} \operatorname{sign}\left(y_{1}-\hat{x}_{1}\right)+\frac{1}{V_{x}}\left(a_{y}-\hat{a}_{y}\right), \\
\hat{a}_{y}=V_{x}\left(A_{21}+1\right) y_{1}+V_{x} A_{22} \hat{x}_{2}+V_{x} B_{2} \delta_{f},
\end{array}\right.
$$

where $c_{1}$ and $c_{2}$ are positive constants.

Then, the following lemma is obtained, whose proof is similar to that in [23]. Thus, it is omitted here.

Lemma 1. The output $\hat{x}_{2}$ of (8) will track $x_{2}$ within a finite time.

Remark 2. It should be pointed out that the values of the front and rear tire cornering stiffness $C_{f}$ and $C_{r}$ are assumed to be constants in the vehicle dynamic model. In fact, their values are dependent on the road condition and normal force on tires, and thus, there are some perturbations for the two parameters. In addition, the model error also exists in the vehicle dynamics. These factors affect the accuracy of the estimation. To fix this problem, the lateral acceleration error $a_{y}-\widehat{a}_{y}$ is introduced in the observer (8) to compensate the model error and parameter perturbations.

\subsection{Baseline Controller}

3.2.1. AFS Controller. The AFS is to control the steering angle of the front wheel in the linear range of the tire. Therefore, the design of the controller is based on 2-DOF. The purpose of the controller is to eliminate the error between the actual vehicle and the ideal vehicle by controlling the front steering angle and to make sure the yaw rate follows the ideal model well.

The active front steering 2-DOF vehicle model is described as

$$
\left[\begin{array}{l}
\dot{r} \\
\dot{\beta}
\end{array}\right]=\left[\begin{array}{ll}
A_{11} & A_{12} \\
A_{21} & A_{22}
\end{array}\right]\left[\begin{array}{l}
r \\
\beta
\end{array}\right]+\left[\begin{array}{l}
B_{1} \\
B_{2}
\end{array}\right] \delta_{f} .
$$

The difference between the actual yaw rate and the ideal value is

$$
e=r-r_{d}
$$

Taking the derivative of (10) yields

$$
\dot{e}=\dot{r}-\dot{r}_{d}
$$

We choose the sliding surface as $s_{1}=e$. Taking the time derivative of $s_{1}$ along system (9) gives

$$
\dot{s}_{1}=A_{11} r+A_{12} \widehat{\beta}+B_{1} \delta_{f}+D_{1}(t),
$$

where $D_{1}=-\dot{r}_{d}+A_{12}(\beta-\widehat{\beta})$. One can find a constant $\gamma_{D_{1}}$ such that

$$
\left|D_{1}(t)\right| \leq \gamma_{D_{1}}
$$

Theorem 1. If the AFS controller is constructed as

$$
\delta_{f}=\frac{1}{B_{1}}\left(-A_{11} r-A_{12} \widehat{\beta}-k_{1} \operatorname{sign}\left(s_{1}\right)-k_{2} s_{1}\right),
$$

where $k_{1}>\gamma_{D_{1}}$ and $k_{2}>0, r$ will finite-time converge to $r_{d}$.

Proof. Putting (14) into (12) yields

$$
\dot{s_{1}}=-k_{1} \operatorname{sign}\left(s_{1}\right)-k_{2} s_{1}+D_{1}(t) \text {. }
$$

Let $V\left(s_{1}\right)=(1 / 2) s_{1}^{2}$. The time derivative of $V\left(s_{1}\right)$ along system (15) is 


$$
\begin{aligned}
\dot{V} & =s_{1} \dot{s}_{1} \\
& =-k_{1} \operatorname{sign}\left(s_{1}\right) s_{1}-k_{2} s_{1}^{2}+D_{1}(t) s_{1} \\
& \leq-k_{1}\left|s_{1}\right|-k_{2} s_{1}^{2}+\left|D_{1}(t)\right|\left|s_{1}\right| \\
& \leq-\left(k_{1}-\gamma_{D_{1}}\right)\left|s_{1}\right|-k_{2} s_{1}^{2} \\
& \leq-\left(k_{1}-\gamma_{D_{1}}\right)\left|s_{1}\right| .
\end{aligned}
$$

Note that $k_{1}>\gamma_{D_{1}}$. It can be proved that $\dot{V} \leq-C V^{1 / 2}$, $C=\sqrt{2}\left(k_{1}-\gamma_{D_{1}}\right)$. From [28-30], the sliding variable $s_{1}$ will finite-time converge to zero. Hence, the corrective steer angle $\Delta \delta_{f}$ generated by the controller is determined as

$$
\Delta \delta_{f}=\delta_{f}-\delta_{f d} .
$$

3.2.2. DYC Controller. DYC control is to utilize the present antilock brake system to obtain the demanded longitudinal force and steady yaw moment. When the yaw moment is taken into account, the equation of state (9) becomes

$$
\begin{aligned}
& \dot{r}=A_{11} r+A_{12} \beta+B_{1} \delta_{f}+\frac{1}{I_{z}} M, \\
& \dot{\beta}=A_{21} r+A_{22} \beta+B_{2} \delta_{f},
\end{aligned}
$$

in which (19) can be rewritten as follows:

$$
\delta_{f}=\frac{1}{B_{2}}\left(\dot{\beta}-A_{21} r-A_{22} \beta\right) .
$$

Substituting (20) into (18) produces

$$
\dot{r}=\left(A_{11}-\frac{B_{1}}{B_{2}} A_{21}\right) r+\left(A_{12}-\frac{B_{1}}{B_{2}} A_{22}\right) \beta+\frac{B_{1}}{B_{2}} \dot{\beta}+\frac{1}{I_{z}} M .
$$

Then, we select a sliding surface, which is composed of the deviation of the yaw rate and the deviation of the sideslip angle, i.e.,

$$
s_{2}=r-r_{d}+\xi\left(\beta-\beta_{d}\right),
$$

with $\xi>0$.

Combining the time derivative of (22) with (21) gives

$$
\dot{s}_{2}=\left(A_{11}-\frac{B_{1}}{B_{2}} A_{21}\right) r+\left(A_{12}-\frac{B_{1}}{B_{2}} A_{22}\right) \hat{\beta}+\frac{1}{I_{z}} M+D_{2}(t),
$$

with $\quad D_{2}(t)=\left(A_{12}-\left(B_{1} / B_{2}\right) A_{22}\right)(\beta-\widehat{\beta})+\left(B_{1} / B_{2}\right) \dot{\beta}+\xi$ $\left(\dot{\beta}-\dot{\beta}_{d}\right)-\dot{r}_{d}$. Note that $\beta$ is often very small, and from the definition of $\beta_{d}$ and $r_{d}, \beta_{d}$ and $\dot{r}_{d}$ are bounded. Hence, a constant $\gamma_{D_{2}}$ can be found such that

$$
\left|D_{2}(t)\right| \leq \gamma_{D_{2}} .
$$

Theorem 2. If the DYC controller is constructed as

$$
\begin{aligned}
M= & I_{z}\left(-\left(A_{11}-\frac{B_{1}}{B_{2}} A_{21}\right) \gamma\right. \\
& \left.-\left(A_{12}-\frac{B_{1}}{B_{2}} A_{22}\right) \widehat{\beta}-K_{1} \operatorname{sign}\left(s_{2}\right)-K_{2} s_{2}\right),
\end{aligned}
$$

where $K_{1}>\gamma_{D_{2}}$ and $K_{2}>0, s_{2}$ will finite-time converge to zero.

Proof. Putting (25) into (23) yields

$$
\dot{s_{2}}=-K_{1} \operatorname{sign}\left(s_{2}\right)-K_{2} s_{2}+D_{2}(t)
$$

Choose the Lyapunov function as $V\left(s_{2}\right)=(1 / 2) s_{2}^{2}$. Differentiating $V\left(s_{2}\right)$ along system (26) gives

$$
\begin{aligned}
\dot{V} & =s_{2}, \\
\dot{s_{2}} & =-K_{1} \operatorname{sign}\left(s_{2}\right) s_{2}-K_{2} s_{2}^{2}+D_{2}(t) s_{2} \\
& \leq-K_{1}\left|s_{2}\right|-K_{2} s_{2}^{2}+\left|D_{2}(t)\right|\left|s_{2}\right| \\
& \leq-\left(K_{1}-\gamma_{D_{2}}\right)\left|s_{2}\right|-K_{2} s_{2}^{2} \\
& \leq-\left(K_{1}-\gamma_{D_{2}}\right)\left|s_{2}\right| .
\end{aligned}
$$

Note that $K_{1}>\gamma_{D_{2}}$. It can be proved that $\dot{V} \leq-C_{\gamma} V^{1 / 2}$, $C_{\gamma}=\sqrt{2}\left(K_{1}-\gamma_{D_{2}}\right)$, and $s_{2}$ finite-time converges to zero.

3.3. Composite Controller Design. In the following, by using DOB technique and baseline controllers, we will give the composite controllers to enhance the control performance.

3.3.1. AFS Controller Based on a DOB. We can rewrite system (12) as

$$
\dot{s_{1}}=F\left(s_{1}\right)+G_{1}\left(s_{1}\right) \delta_{f}+G_{2}\left(s_{1}\right) D_{1}(t),
$$

where $F\left(s_{1}\right)=A_{11} r+A_{12} \widehat{\beta}, G_{1}\left(s_{1}\right)=B_{1}, G_{2}\left(s_{1}\right)=1$, and $D_{1}(t)$ is regarded as the unknown disturbance and satisfies $\left|\dot{D}_{1}(t)\right| \leq \gamma_{\dot{D}_{1}}$ with $\gamma_{\dot{D}_{1}}>0$ being a constant, which at least holds locally.

Design the nonlinear DOB (NDOB) as

$$
\left\{\begin{array}{l}
\dot{P}=-L_{1} G_{2} P-L_{1}\left[G_{2} L_{1} s_{1}+F\left(s_{1}\right)+G_{1} \delta_{f}\right], \\
D_{1}=\widehat{P}+L_{1} s_{1},
\end{array}\right.
$$

where $P$ and $L_{1}$ are the state and constant.

Let $e_{1}(t)=D_{1}(t)-\widehat{D}_{1}(t)$. Differentiating $e_{1}(t)$ along systems (28) and (29) gives

$$
\begin{aligned}
\dot{e}_{1} & =\dot{D}_{1}-\dot{\hat{D}}_{1} \\
& =\dot{D}_{1}-\left[-L_{1} G_{2} P-L_{1}^{2} G_{2} s_{1}+L_{1} G_{2} D_{1}\right] \\
& =\dot{D}_{1}-L_{1} G_{2} e_{1} .
\end{aligned}
$$

Let $V\left(e_{1}\right)=(1 / 2) e_{1}^{2}$. Then, one has

$$
\dot{V}\left(e_{1}\right)=e_{1} \dot{e}_{1}=e_{1}\left(\dot{D}_{1}-L_{1} G_{2} e_{1}\right) \leq\left|e_{1}\right| \gamma_{\dot{D}_{1}}-L_{1} G_{2} e_{1}^{2} \text {. }
$$


Define a region as $Q_{1}=\left\{e_{1}:\left|e_{1}\right| \leq\left(\gamma_{\dot{D}_{1}} / L_{1} G_{2}\right)\right\}$. For any $e_{1}(t) \in R / Q_{1}$, one has $\left|e_{1}\right|>\left(\gamma_{\dot{D}_{1}} / L_{1} G_{2}\right)$. This, together with (31), yields $\dot{V}\left(e_{1}\right) \leq-\left|e_{1}\right|\left(L_{1} G_{2}\left|e_{1}\right|-\gamma_{\dot{D}_{1}}\right)<0$. Note that $G_{2}=1$, which means that $e_{1}(t)$ will reach and stay in the domain

$$
Q_{1}=\left\{e_{1}:\left|e_{1}\right| \leq \frac{\gamma_{\dot{D}_{1}}}{L_{1}}\right\}
$$

Theorem 3. Provided that the composite AFS controller is constructed as

$$
\delta_{f}=\frac{1}{B_{1}}\left(-A_{11} r-A_{12} \widehat{\beta}-\widehat{D}_{1}-k_{1} \operatorname{sign}\left(s_{1}\right)-k_{2} s_{1}\right),
$$

where $k_{1}>0, k_{2}>0$, and $L_{1}>\gamma_{\dot{D}_{1}}, s_{1}$ will finite-time converge to zero.

Proof. Putting (33) into (12) yields

$$
\begin{aligned}
\dot{s_{1}} & =-K_{1} \operatorname{sign}\left(s_{1}\right)-K_{2} s_{1}+D_{1}(t)-\widehat{D}_{1} \\
& =-K_{1} \cdot \operatorname{sign}\left(s_{1}\right)-K_{2} s_{1}+e_{1}(t) .
\end{aligned}
$$

From NDOB (29), it is clear that the error $e_{1}(t)$ is bounded. This means that a constant $\gamma_{e_{1}}$ can be found such that $\left|e_{1}(t)\right|=\left|D_{1}(t)-\widehat{D}_{1}\right| \leq \gamma_{e_{1}}$.

Hence, the corrective steer angle $\Delta \delta_{f}$ generated by the controller is determined as follows:

$$
\Delta \delta_{f}=\delta_{f}-\delta_{f d} .
$$

3.3.2. DYC Controller Based on a DOB. System (23) can be rewritten as

$$
\dot{s}_{2}=f\left(s_{2}\right)+g_{1}\left(s_{2}\right) M+g_{2}\left(s_{2}\right) D_{2}(t),
$$

where $f\left(s_{2}\right)=\left(A_{11}-\left(B_{1} / B_{2}\right) A_{21}\right) r+\left(A_{12}-\left(B_{1} / B_{2}\right) A_{22}\right) \widehat{\beta}$, $g_{1}\left(s_{2}\right)=1 / I_{z}, g_{2}\left(s_{2}\right)=1$, and $D_{2}(t)$ is considered as the unknown perturbation, and $\left|\dot{D}_{2}(t)\right| \leq \gamma_{\dot{D}_{2}}$. Then, a NDOB is constructed as

$$
\left\{\begin{array}{l}
\dot{p}=-L_{2} g_{2} p-L_{2}\left[g_{2} L_{2} s_{2}+f\left(s_{2}\right)+g_{1} M\right], \\
\widehat{D_{2}}=p+L_{2} s_{2} .
\end{array}\right.
$$

$L_{2}$ is a positive constant.

Let $e_{2}(t)=D_{2}(t)-\widehat{D}_{2}(t)$. Taking the derivative of $e_{2}(t)$ along systems (36) and (37) gives

$$
\begin{aligned}
\dot{e}_{2} & =\dot{D}_{2}-\dot{\vec{D}}_{2} \\
& =\dot{D}_{2}-\left[-L_{2} g_{2} p-L_{2}^{2} g_{2} s_{2}+L_{2} g_{2} D_{2}\right] \\
& =\dot{D}_{2}-L_{2} g_{2} e_{2} .
\end{aligned}
$$

We choose a Lyapunov function as $V\left(e_{2}\right)=(1 / 2) e_{2}^{2}$, whose time derivative along (38) is

$$
\dot{V}\left(e_{2}\right)=e_{2} \dot{e}_{2}=e_{2}\left(\dot{D}_{2}-L_{2} g_{2} e_{2}\right) \leq\left|e_{2}\right| \gamma_{\dot{D}_{2}}-L_{2} g_{2} e_{1}^{2} \text {. }
$$

Define a region as $Q_{2}=\left\{e_{2}:\left|e_{2}\right| \leq \gamma_{\dot{D}_{2}} / L_{2} g_{2}\right\}$. For any $e_{2}(t) \in R / Q_{2}$, we have $\left|e_{2}\right|>\gamma_{\dot{D}_{2}} / L_{2} g_{2}$. This, together with (39), yields $\dot{V}\left(e_{2}\right) \leq-\left|e_{2}\right|\left(L_{2} g_{2}\left|e_{2}\right|-\gamma_{\dot{D}_{2}}\right)<0$. It is noted that $g_{2}=1$. This means that $e_{2}(t)$ will stay in the domain

$$
Q_{2}=\left\{e_{2}:\left|e_{2}\right| \leq \frac{\gamma_{\dot{D}_{2}}}{L_{2}}\right\} .
$$

Theorem 4. Provided that the composite DYC controller is constructed as

$$
\begin{aligned}
M= & I_{z}\left(-\left(A_{11}-\frac{B_{1}}{B_{2}} A_{21}\right) r-\left(A_{12}-\frac{B_{1}}{B_{2}} A_{22}\right) \widehat{\beta}\right. \\
& \left.-K_{1} \operatorname{sign}\left(s_{2}\right)-K_{2} s_{2}-\widehat{D_{2}}\right),
\end{aligned}
$$

where $k_{1}>0, k_{2}>0$, and $L_{1}>\gamma_{\dot{D}_{1}}, s_{2}$ will finite-time converge to zero.

Proof. Putting (41) into (23) yields

$$
\begin{aligned}
\dot{s_{2}} & =-k_{1} \operatorname{sign}\left(s_{2}\right)-k_{2} s_{2}+D_{2}(t)-\widehat{D}_{2} \\
& =-k_{1} \cdot \operatorname{sign}\left(s_{2}\right)-k_{2} s_{2}+e_{2}(t) .
\end{aligned}
$$

From NDOB (37), it is clear that the error $e_{2}(t)$ is bounded. This means that a constant $\gamma_{e_{2}}>0$ can be found such that $\left|e_{2}(t)\right|=\left|D_{2}(t)-\widehat{D}_{2}\right| \leq \gamma_{e_{2}}$. The remainder of the proof is similar to that in Theorem 2 .

3.4. Torque Distribution Controller. On the basis of the ideal yaw moment obtained by the DYC controller, the main function of the whole control strategy is to assign the expected yaw moment calculated by the DYC controller to four wheels. The torque distributor designed in this paper is based on the vertical load distribution, taking into account the motor output and road surface conditions of the distribution constraints. First of all, the relationship between the longitudinal force of the tire and the torque of the motor is

$$
F_{x i j}=\frac{T_{i j}}{R},
$$

which leads to

$$
\begin{aligned}
T_{f l} & =\frac{F_{z f l}}{F_{z}} \frac{M_{z}}{-d_{f} / 2 \cos \delta_{f d}+a \sin \delta_{f d}} R, \\
T_{f r} & =\frac{F_{z f r}}{F_{z}} \frac{M_{z}}{d_{f} / 2 \cos \delta_{f d}+a \sin \delta_{f d}} R, \\
T_{r l} & =-\frac{F_{z r l}}{F_{z}} \frac{M_{z}}{2 d_{r}} R, \\
T_{f l} & =\frac{F_{z r r}}{F_{z}} \frac{M_{z}}{2 d_{r}} R .
\end{aligned}
$$

Finally, the torques obtained by formula (44) are constrained as follows: 
TABle 1: Parameters of the vehicle model.

\begin{tabular}{lc}
\hline Symbol & Value \\
\hline$m$ & $1429(\mathrm{Kg})$ \\
$b$ & $1.569(\mathrm{~m})$ \\
$d_{r}$ & $1.565(\mathrm{~m})$ \\
$I_{z}$ & $1765\left(\mathrm{Kg} \cdot \mathrm{m}^{2}\right)$ \\
$C_{r}$ & $87002(\mathrm{~N} / \mathrm{rad})$ \\
$a$ & $1.05(\mathrm{~m})$ \\
$d_{f}$ & $1.565(\mathrm{~m})$ \\
$R$ & $0.35(\mathrm{~m})$ \\
$C_{f}$ & $79240(\mathrm{~N} / \mathrm{rad})$ \\
$n$ & 20 \\
\hline
\end{tabular}

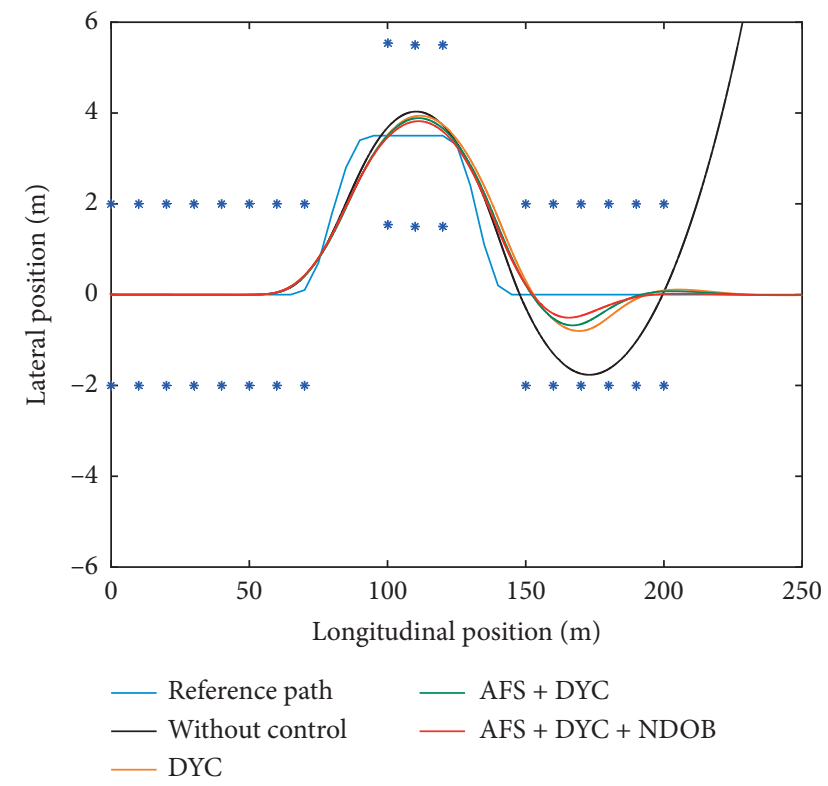

FIgURE 3: The vehicle trajectory.

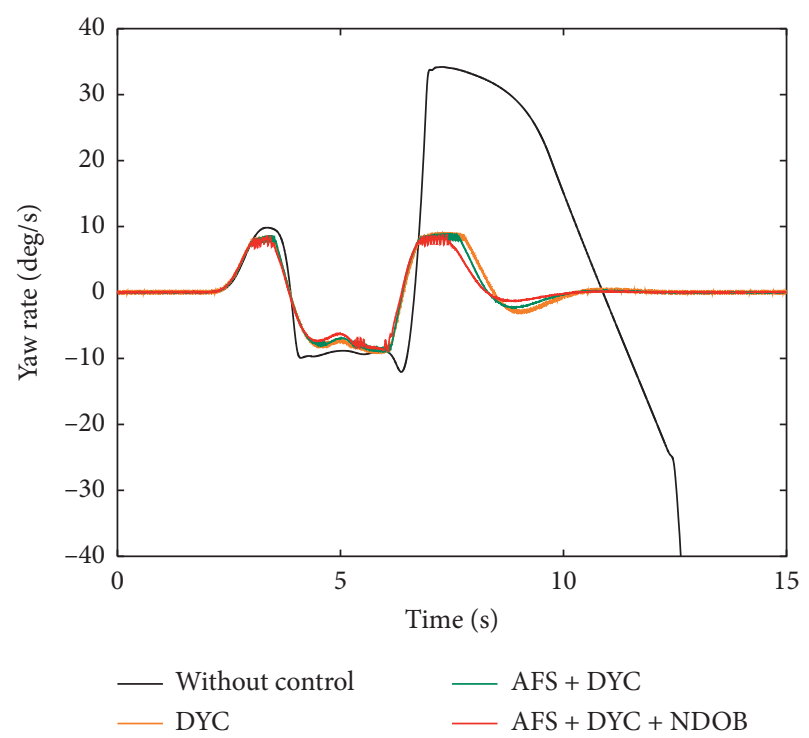

Figure 4: Time history of the yaw rate.

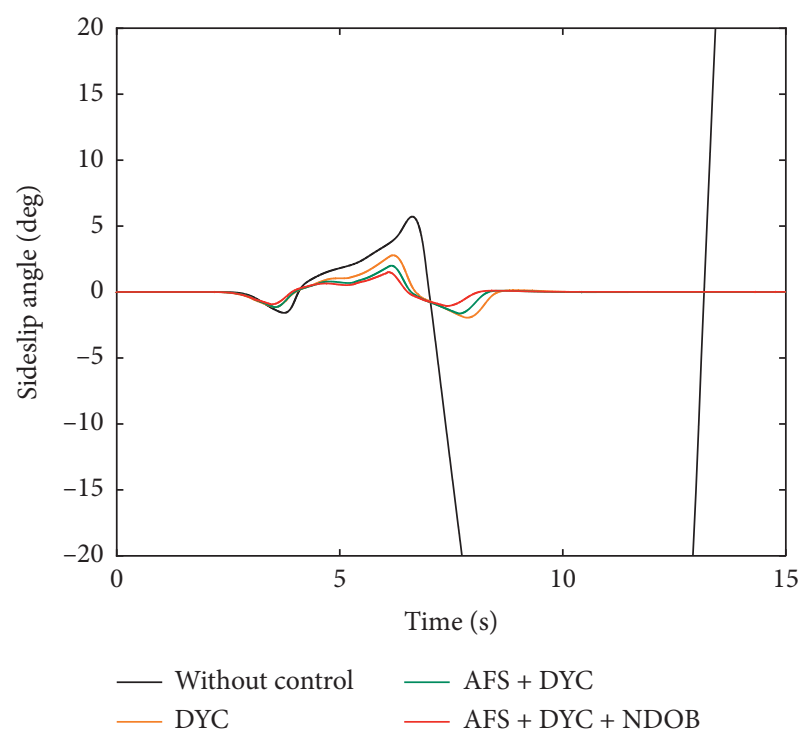

Figure 5: Time history of the sideslip angle.

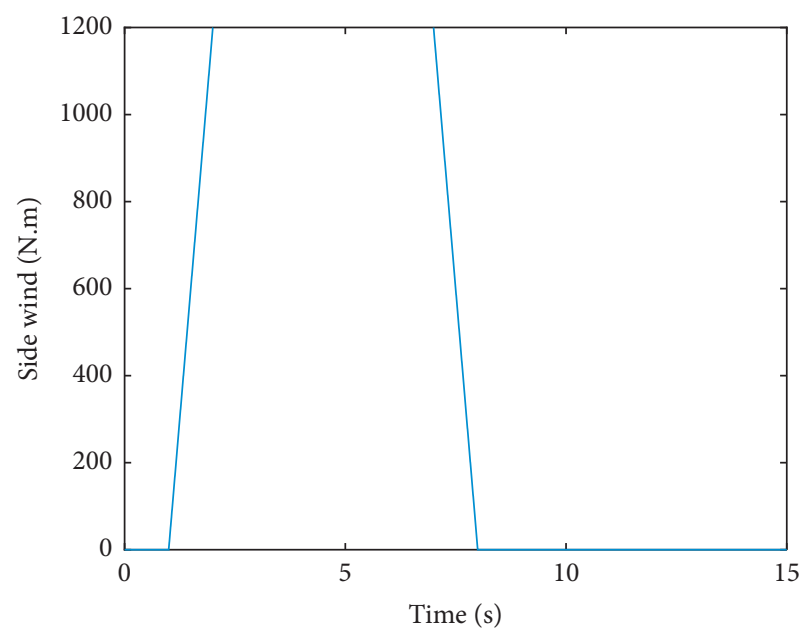

Figure 6: The side wind disturbance input.

$$
\left|T_{i j}\right| \leq \min \left(\mu R F_{z i j}, T_{\max }\right),
$$

where $F_{z i j}$ is the driving or braking torque, $T_{i j}$ is the vertical load, $T_{\text {max }}$ is the maximum motor output torque, $\mu$ is the adhesion coefficient of the road, and $R$ represents the effective radius of the tire.

\section{Simulation Results}

The validity of the AFS and DYC control is shown by using the cosimulation of MATLAB/Simulink and CarSim. The responses are compared with those without any control, with yaw-moment control only, and with the AFS and DYC integrated control. The parameters are given in Table 1.

In the simulation, the initial speed of the vehicle is $80 \mathrm{~km} / \mathrm{h}$, and $\mu$ is 0.3 in the double lane-changing maneuver, and $\xi=0.5$. 


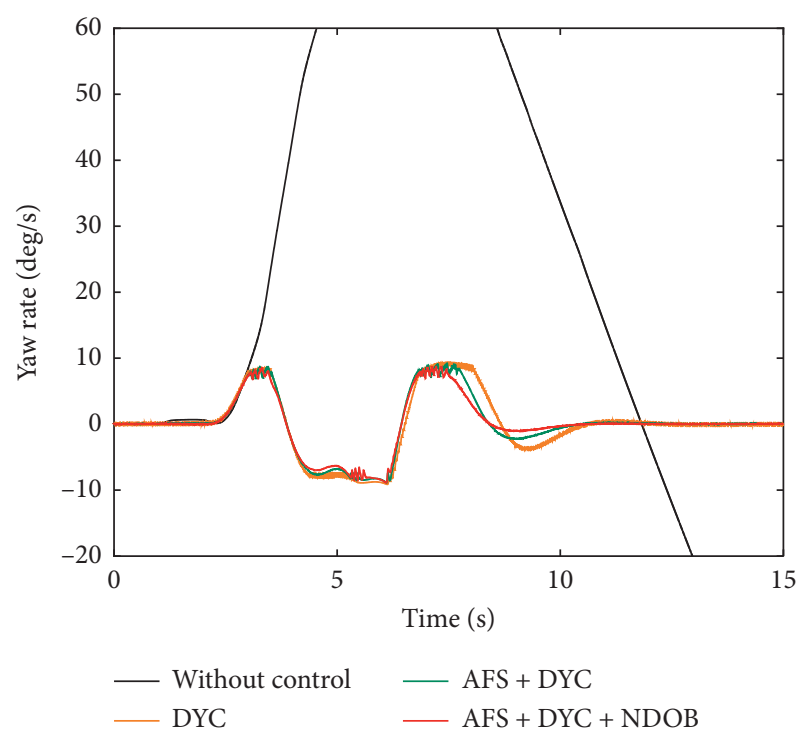

Figure 7: The response curve of the yaw rate.

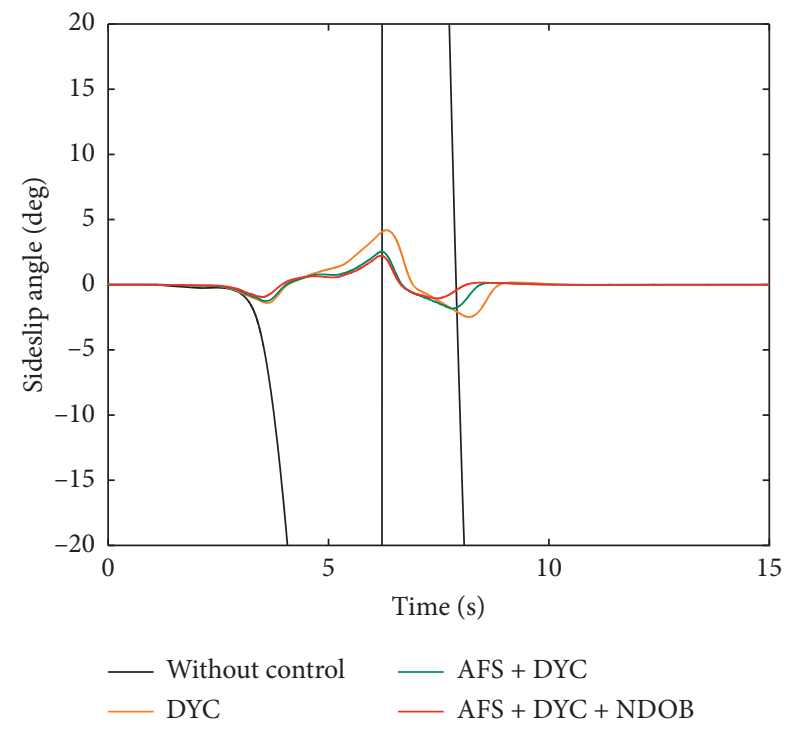

FIgUre 8: Time history of the sideslip angle.

4.1. Double Lane-Change Maneuver. The simulation results are depicted in Figures 3-5. It can be seen from Figures 4 and 5 that, without any control, the yaw rate and the sideslip angle are larger values deviating from their ideal value, even cannot guarantee the stability of the vehicle. On the contrary, under the other three controllers, the vehicle trajectory can track the expected trajectory, the actual yaw rate can follow the ideal value, and the actual sideslip angle can be controlled in a stable region. Compared with other controllers, the integrated control of AFS and DYC based on the DOB technique can improve the vehicle response, which can also be verified by Figure 3 .

4.2. Response to Side Wind Disturbance. The simulation is given under a side wind force, as shown in Figure 6. It can be seen from Figures 7 and 8 that the case without any control

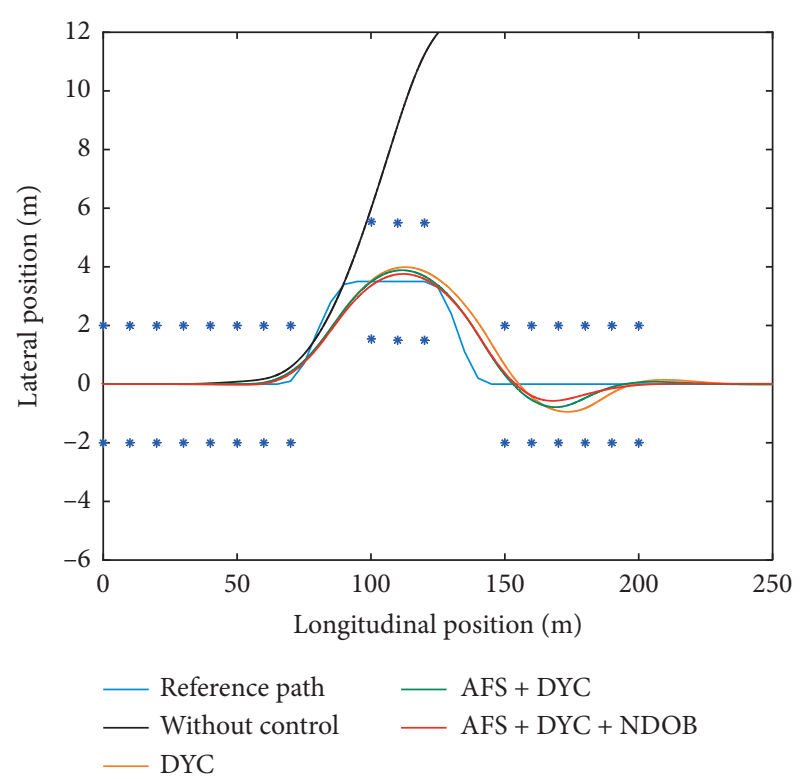

FIgURE 9: The vehicle trajectory.

fails to keep vehicle's stability, while the case with the yawmoment control only cannot control well compared with the integrated control. The properties can be clearly seen in Figure 9, which reflects the double lane-change maneuvering under the proposed controllers. At last, it can also be observed from Figure 9 that the integrated control of AFS and DYC based on the DOB technique has better control performance compared with the pure AFS and DYC integrated control.

\section{Conclusion}

In our study, it has been shown that the AFS and DYC integrated control has better control performance than the DYC control. At the same time, we also confirm that the integrated control of AFS and DYC based on the DOB technique can suppress the large disturbance and have better robustness in comparison with the pure AFS and DYC integrated control [31].

\section{Data Availability}

No data were used to support this study.

\section{Conflicts of Interest}

The authors declare that there are no conflicts of interest.

\section{Acknowledgments}

This work was supported by the Guiding Funds for Industrial Development of Suqian City under grant S201920.

\section{References}

[1] A. Zanten, R. Erhardt, and G. Pfaff, "The vehicle dynamics control system of bosch," 1995. SAE Technical Paper, Article ID 950759.

[2] D. Zhang, Y.-P. Shen, S.-Q. Zhou, X.-W. Dong, and L. Yu, "Distributed secure platoon control of connected vehicles 
subject to DoS attack: theory and application," IEEE Transactions on Systems, Man, and Cybernetics: Systems, p. 1, 2020.

[3] Z. Xu, H. Ni, H. Reza Karimi, and D. Zhang, "A Markovian jump system approach to consensus of heterogeneous multiagent systems with partially unknown and uncertain attack strategies," International Journal of Robust and Nonlinear Control, vol. 30, no. 7, pp. 3039-3053, 2020.

[4] C. Doniselli, G. Mastinu, and R. Cal, "Traction control for front-wheel-drive vehicles," Vehicle System Dynamics, vol. 23, no. sup1, pp. 87-104, 1994.

[5] D. V. T. Truong and W. Tomaske, "Active front steering system using adaptive sliding mode control," in Proceedings of the Control and Decision Conference, vol. 253-258, IEEE, Firenze, Italy, 2013.

[6] Y. Shibahata, K. Shimada, and T. Tomari, "Improvement of vehicle maneuverability by direct yaw moment control," Vehicle System Dynamics, vol. 22, no. 5-6, pp. 465-481, 1993.

[7] M. Nagai, M. Shino, and F. Gao, "Study on integrated control of active front steer angle and direct yaw moment," Jsae Review, vol. 23, no. 3, pp. 309-315, 2002.

[8] W. Yao, W. U. Maosheng, and Y. U. Datai, "A method to improve braking stability by the integrated control of yawmoment control and active front steering," Journal of University of Science Technology Beijing, vol. 4, no. 4, 2005.

[9] X. Gao and Z. A. Yu, "Coordinated control of AFS and ESP based on vehicle state identification," International Journal of Vehicle Mechanics and Mobility, vol. 47, 2007.

[10] X. N. Wen and W. H. Chai, AFS/DYC Integrated Control Strategy Research Based on Fuzzy Logic, Changchun University, Changchun, China, 2009.

[11] G. Li, C. F. Zong, and L. Y. Jiang, "Active front steering and direct yaw moment integrated control algorithm," Vehicle System Dynamics, vol. 48, 2011.

[12] L. Liu, S. Ding, and X. Yu, "Second-order sliding mode control design subject to an asymmetric output constraint," IEEE Transactions on Circuits and Systems II: Express Briefs, vol. 112, p. 1, 2020.

[13] Q. Hou, S. Ding, and X. Yu, "Composite super-twisting sliding mode control design for PMSM speed regulation problem based on a novel disturbance observer," IEEE Transactions on Energy Conversion, p. 1, 2020.

[14] Q. Hou and S. Ding, "GPIO based super-twisting sliding mode control for PMSM," IEEE Transactions on Circuits and Systems II: Express Briefs, p. 1, 2020.

[15] L. Liu, W. X. Zheng, and S. Ding, "An adaptive SOSM controller design by using a sliding-mode-based filter and its application to Buck converter," IEEE Transactions on Circuits and Systems I: Regular Papers, vol. 67, no. 7, pp. 2409-2418, 2020.

[16] S. Ding, J. H. Park, and C. C. Chen, "Second-order sliding mode controller design with output contraint," Automatica, vol. 112, Article ID 108704, 2020.

[17] Q. Meng, C. Qian, and R. Liu, "Dual-rate sampled-data stabilization for active suspension system of electric vehicle," International Journal of Robust and Nonlinear Control, vol. 28, no. 5, pp. 1610-1623, 2018.

[18] S. Ding, W. Chen, K. Mei, and D. Murray-Smith, "Disturbance observer design for nonlinear systems represented by input-output models," IEEE Transactions on Industrial Electronics, vol. 67, no. 2, pp. 1222-1232, 2019.

[19] H. Du, C. Jiang, G. Wen, W. Zhu, and Y. Cheng, "Current sharing control for parallel DC-DC Buck converters based on finite-time control technique," IEEE Transactions on Industrial Informatics, vol. 15, no. 4, pp. 2186-2198, 2019.
[20] Q. Meng, T. Zhao, C. Qian, Z. Sun, and P. Ge, "Integrated stability control of AFS and DYC for electric vehicle based on non-smooth control," International Journal of Systems Science, vol. 49, no. 7, pp. 1518-1528, 2019.

[21] A. Mehmet and J. Kalkkuhl, "Lateral dynamics emulation via a four-wheel steering vehicle," Vehicle System Dynamics, vol. 46, no. 9, pp. 803-829, 2008.

[22] Q. Zhou, F. Wang, and L. Li, "Robust sliding mode control of 4WS vehicles for automatic path tracking," in Proceedings of Intelligent Vehicles Symposium, pp. 819-826, IEEE, Vegas, NV, USA, 2005.

[23] S. Ding, L. Liu, and W. X. Zheng, "Sliding mode direct yawmoment control design for in-wheel electric vehicles," IEEE Transactions on Industrial Electronics, vol. 64, no. 8, pp. 6752-6762, 2017.

[24] W. H. Chen, "Nonlinear disturbance observer-enhanced dynamic inversion control of missiles," Journal of Guidance Control Dynamics, vol. 26, no. 1, pp. 161-166, 2015.

[25] Y. Ji, H. Guo, and H. Chen, "Integrated control of active front steering and direct yaw moment based on model predictive control," in Proceedings of Control and Decision Conference, IEEE, Nanchang, China, 2014.

[26] S. Zhang, S. Ding, and H. Jiang, "Direct yaw-moment control of in-wheel electric vehicle by sliding mode technique," in Proceedings of Conference on Industrial Technology, IEEE, Nanchang, China, 2016.

[27] U. Kiencke and L. Nielsen, Automotive Control Systems, Springer, Berlin, Germany, 2005.

[28] Z.-Y. Sun, Y. Shao, and C.-C. Chen, "Fast finite-time stability and its application in adaptive control of high-order nonlinear system," Automatica, vol. 106, no. 5, pp. 339-348, 2019.

[29] Z. Sun, Y. Shao, C. Chen, and Q. Meng, "Global outputfeedback stabilization for stochastic nonlinear systems: a double-domination approach," International Journal of Robust and Nonlinear Control, vol. 28, no. 5, pp. 4635-4646, 2018.

[30] C. Chen and Z. Sun, "A unified approach to finite-time stabilization of high-order nonlinear systems with an asymmetric output constraint," Automatica, vol. 111, Article ID 108581, 2020. 